\title{
Tuning Colloid-Interface Interactions by Salt Partitioning
}

\author{
J. C. Everts, ' S. Samin, and R. van Roij \\ Institute for Theoretical Physics, Center for Extreme Matter and Emergent Phenomena, Utrecht University, \\ Princetonplein 5, 3584 CC Utrecht, The Netherlands
}

(Received 29 March 2016; published 23 August 2016)

\begin{abstract}
We show that the interaction of an oil-dispersed colloidal particle with an oil-water interface is highly tunable from attractive to repulsive, either by varying the sign of the colloidal charge via charge regulation or by varying the difference in hydrophilicity between the dissolved cations and anions. In addition, we investigate the yet unexplored interplay between the self-regulated colloidal surface charge distribution with the planar double layer across the oil-water interface and the spherical one around the colloid. Our findings explain recent experiments and have direct relevance for tunable Pickering emulsions.
\end{abstract}

DOI: 10.1103/PhysRevLett.117.098002

Colloidal particles experience a deep potential well when they intersect fluid-fluid interfaces. They therefore adsorb strongly to such interfaces, self-assembling into structures such as two-dimensional monolayers [1] or particle-laden droplets in Pickering emulsions [2,3]. The free energy gain caused by the reduction of the fluid-fluid surface area is $\gamma \pi a^{2}(1+\cos \theta)^{2} \simeq 10^{3}-10^{7} k_{B} T$, where $\gamma$ is the fluid-fluid surface tension, the particle radius $a$ is typically between 10 and $10^{3} \mathrm{~nm}, \theta$ is the three-phase contact angle, and $k_{B} T$ is the thermal energy [1]. With the exception of nanoparticles $[4,5]$, the binding is essentially irreversible and hardly prone to physicochemical modifications such as the $p \mathrm{H}$ or salt concentration. Only strong mechanical agitation is able to detach micron-sized particles from the interface [6]. However, the recovery of particles from fluid-fluid interfaces is an essential step for the realization of applications, such as in biofuel upgrade [7], "dry water" catalysis [8], and gas storage [9].

An alternative route to overcome the difficulties associated with strong adsorption was offered in Refs. [3,10], which show that charged poly(methylmethacrylate) (PMMA) particles that stabilize water-cyclochexylbromide (CHB) Pickering emulsions are (almost) nonwetting $(\cos \theta \rightarrow-1)$, such that the colloidal particles reside essentially in the oil phase. The crucial ingredient of this system is the relatively "polar" oil which solvates a small but significant amount of charge that stabilizes the colloids [3,11]. Within a modified Poisson-Boltzmann theory, qualitative agreement was found with the experimental out-of-plane structure of the particles, provided a small degree of wetting was assumed $[10,12]$.

Very recent experiments on the same system by Elbers et al. [13] revealed that the colloidal particles in fact do not penetrate the water-CHB interface but are trapped at a finite $\sim$ nanometer distance $[13,14]$, completely circumventing the irreversible wetting effects described above. Additionally, these nontouching particles were easily detached from the oilwater interface by the addition of an organic salt $[13,15]$ while at the same time reversing the sign of the colloidal charge; see
Ref. [13]. Interestingly, this tunability offers an appealing route for the controlled destabilization of Pickering emulsions. The authors of Ref. [13] hypothesized that the trapping mechanism is due to a force balance between an attractive image-charge and a repulsive van der Waals (VDW) force, as proposed by Oettel [16]. While correctly predicting the nontouching behavior, this argument cannot explain how the particle-interface interaction can be tuned from attractive to long-ranged repulsive by adding salt.

The findings of Ref. [13] show that whether a particle ever arrives at the interface is subtle and tunable. In this Letter, we show that the interaction between a charged colloidal particle and the oil-water interface consists not only of well-known image-charge $[17,18]$ and dispersion forces [16,19], but also of particle-ion forces that can be tuned between strongly repulsive and strongly attractive by (i) varying the sign of the particle charge and (ii) by varying the difference between the degree of hydrophilicity of the cations and anions. This difference determines not only the distribution of ions among the water and oil phases ("ion partitioning"), but also the sign of the Donnan potential that spontaneously forms between the water and oil bulk phases (not unlike the potential at a $P N$ junction) $[10,20-24]$. In fact, our calculations show contrary to a common assumption [16,25-27] that the low but nonzero ion concentrations in oil are crucial and should not be neglected [28-34], even if the ions initially present in the oil strongly prefer to be in the water phase [20-24,35-42].

To describe the coupling between the particle, oil-water interface, and ions, we will focus for simplicity on a single oildispersed charged colloidal sphere of radius $a=1 \mu \mathrm{m}$, charge $Z e$, and dielectric constant $\epsilon_{c}=2.6$ (PMMA) with its center at a distance $d$ from a planar oil-water interface [43] that separates the oil (CHB) phase $(z>0$, dielectric constant $\left.\epsilon_{o}=7.92\right)$ from the water phase $(z<0$, dielectric constant $\epsilon_{w}=80$ ), as sketched in Fig. 1(a). Since $\epsilon_{c}<\epsilon_{o}<\epsilon_{w}$, the VDW interaction between the particle and the oil-water interface is repulsive $[16,19]$ for $d>a$. This repulsion is significant only for $d-a \lesssim 10 \mathrm{~nm}$ given the Hamaker 

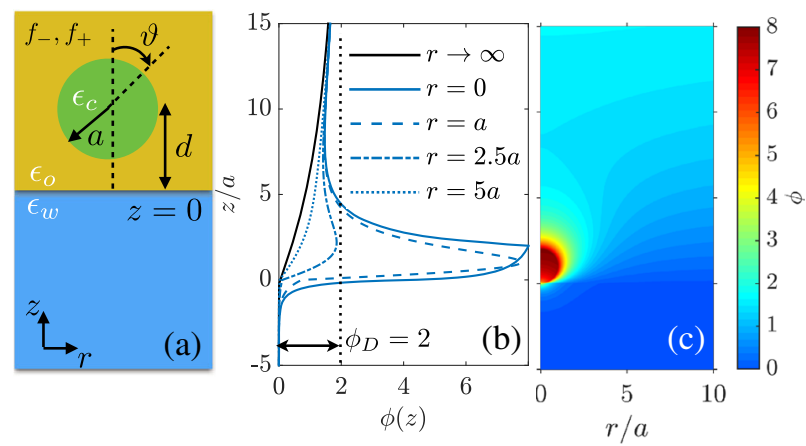

FIG. 1. (a) Geometry of a colloidal sphere with radius $a=1 \mu \mathrm{m}$ and a dielectric constant $\epsilon_{c}=2.6$ in cylindrical coordinates $(r, z)$ at a distance $d$ from an oil-water interface, with the position on the particle surface indicated by the polar angle $\vartheta$. The oil and water are characterized by dielectric constants $\epsilon_{o}=7.92$ and $\epsilon_{w}=80$, respectively. The self-energies $\left(f_{+}, f_{-}\right)=(6,10)$ in units of $k_{B} T$ determine the degree of ion partitioning among the two solvents. In (b), we show the electrostatic potential $\phi(r, z) / \beta e$ for a positively charged sphere at $d=a$ along the $z$ direction at fixed $r$, with the Donnan potential $\phi_{D}$ indicated, and as a surface plot in (c). We set the charge-regulating equilibrium constant $K_{+} a^{3}=1$ $\left(p K_{+}=8.8\right)$ and the screening length in oil $\kappa_{o}^{-1}=10 \mu \mathrm{m}$.

constant of $\sim-0.3 k_{B} T$ [13]. However, the repulsion is sufficiently strong to impede adsorption to the interface [16]; see Ref. [45]. Since $\epsilon_{o}<\epsilon_{w}$, the image charge potential $\Phi_{\mathrm{im}}(d)=-\beta^{-1} Z^{2} \lambda_{B}^{o}\left(\epsilon_{w}-\epsilon_{0}\right) /\left[4 d\left(\epsilon_{w}+\epsilon_{0}\right)\right]\left(\right.$ where $\beta^{-1}=$ $k_{B} T$ ), which holds in the absence of salt for $\epsilon_{c}=\epsilon_{o}$ but which was shown to be accurate within a few percent also for nonindex-matched colloidal particles [25], is attractive regardless the sign of $Z$ for oil-dispersed colloidal particles but is repulsive when the particles are dispersed in the water phase [18]. Here, $\lambda_{B}^{o}=\beta e^{2} / 4 \pi \epsilon_{\mathrm{vac}} \epsilon_{o}$ is the Bjerrum length in oil. A force balance between the image and VDW force shows that it is possible to trap the particle at a finite, but small, distance from the interface $[13,16]$, circumventing the nontunable nature of the wetting effects. Incorporating the VDW force in our theory (unlike Ref. [10]), we show how screening and ion partitioning makes the trapping mechanism highly tunable.

We use the framework of the classical density-functional theory to construct the grand potential functional $\Omega\left[\rho_{ \pm}, \sigma ; d\right]$, with $\rho_{ \pm}(\mathbf{r})$ the density profiles of (nonsurface-bound) monovalent ions and $\sigma(\mathbf{r})$ the particle surface charge density. Denoting the region outside the particle by $\mathcal{R}$ and its surface by $\Gamma, \Omega$ is given by

$$
\begin{aligned}
\beta \Omega= & \sum_{\alpha} \int_{\mathcal{R}} d^{3} \mathbf{r} \rho_{\alpha}(\mathbf{r})\left[\ln \left(\frac{\rho_{\alpha}(\mathbf{r})}{\rho_{s}^{w}}\right)-1+\beta V_{\alpha}(z)\right] \\
& +\int_{\Gamma} d^{2} \mathbf{r}\left( \pm \sigma(\mathbf{r})\left\{\ln \left[ \pm \sigma(\mathbf{r}) a^{2}\right]+\ln \left(\frac{K_{ \pm}}{\rho_{s}^{w}}\right)+\beta V_{ \pm}(z)\right\}\right. \\
& \left.+\left[\sigma_{m} \mp \sigma(\mathbf{r})\right] \ln \left\{\left[\sigma_{m} \mp \sigma(\mathbf{r})\right] a^{2}\right\}\right)+\frac{1}{2} \int_{\mathcal{R}} d^{3} \mathbf{r} Q(\mathbf{r}) \phi(\mathbf{r}) .
\end{aligned}
$$

The first term is the ideal gas grand potential of the ions coupled to the external potential $V_{\alpha}(z)=\beta^{-1} f_{\alpha} \Theta(z)$ $(\alpha= \pm)$, with $\Theta(z)=[1+\tanh (z / 2 \xi)] / 2, \xi \sim 10^{-3} a$ the interface thickness, and $\rho_{s}^{w}$ the bulk density in water. The preference of ions for water or oil is modeled by the energy cost $\beta^{-1} f_{\alpha}$ to transfer a single ion from the water to the oil phase. To mimic that cations are typically less hydrophilic than anions, we set $\left(f_{+}, f_{-}\right)=(6,10)$. The second term describes the free energy of a two-dimensional binary lattice gas of neutral and (either positively or negatively) charged groups, with a maximum charge density $\sigma_{m} a^{2}=10^{6}$ (one charged group per $\mathrm{nm}^{2}$ ). The nonelectrostatic free energy of binding an ion is characterized by $k_{B} T \ln \left(K_{ \pm} / 1 \mathrm{M}\right)$, with equilibrium constant $K_{ \pm}=[S]\left[X^{ \pm}\right] /\left[S X^{ \pm}\right]$[or $p K_{ \pm}=$ $\left.-\log _{10}\left(K_{ \pm} / 1 \mathrm{M}\right)\right]$ that describes the adsorption of a negative or positive ion $X^{ \pm}$to a neutral surface site $S$, i.e., $S+X^{ \pm} \leftrightarrows S X^{ \pm}$. The electrostatic energy is described within mean-field theory in the last term, with the total charge density $Q(\mathbf{r})=\rho_{+}(\mathbf{r})-\rho_{-}(\mathbf{r})+\sigma(\mathbf{r}) \delta\left(\left|\mathbf{r}-d \mathbf{e}_{z}\right|-a\right)$, and the electrostatic potential $\phi(\mathbf{r}) / \beta e=25.6 \phi(\mathbf{r}) \mathrm{mV}$.

From the Euler-Lagrange equations $\delta \Omega / \delta \rho_{ \pm}(\mathbf{r})=0$, we find the equilibrium profiles $\rho_{ \pm}(\mathbf{r})=\rho_{s}(z) \exp [\mp \phi(\mathbf{r}) \pm$ $\left.\Theta(z) \phi_{D}\right]$, where $\rho_{s}(z)=\rho_{s}^{w}$ for $z<0$ and $\rho_{s}(z)=\rho_{s}^{o}$ for $z>0$, where $\rho_{s}^{o}=\rho_{s}^{w} \exp \left[-\left(f_{+}+f_{-}\right) / 2\right]$ the bulk ion density in oil. We defined the Donnan potential $\phi_{D} / \beta e$, with $\phi_{D}=\left(f_{-}-f_{+}\right) / 2$, which is the potential difference between the bulk oil and water phases due to ion partitioning. Combining our expressions for $\rho_{ \pm}(\mathbf{r})$ with the Poisson equation for the electrostatic potential, we obtain the Poisson-Boltzmann equation for $\mathbf{r} \in \mathcal{R}$ :

$$
\nabla \cdot[\epsilon(z) \nabla \phi(\mathbf{r})] / \epsilon_{o}=\kappa(z)^{2} \sinh \left[\phi(\mathbf{r})-\Theta(z) \phi_{D}\right],
$$

where $\epsilon(z)=\left(\epsilon_{o}-\epsilon_{w}\right) \Theta(z)+\epsilon_{w}$. Furthermore, $\kappa(z)^{2}=$ $8 \pi \lambda_{B}^{o} \rho_{s}(z)$, with $\kappa^{-1}(z \rightarrow \infty)$ the Debye screening length in the bulk oil $\kappa_{o}^{-1}$. We fix $\kappa_{o}^{-1}=10 a$, close to typical values in CHB [34], from which the screening length in water follows as $\kappa_{w}^{-1}=\sqrt{\epsilon_{w} / \epsilon_{o}} \exp \left[-\left(f_{+}+f_{-}\right) / 4\right] \kappa_{o}^{-1}=0.58 a$, which is on the high side but convenient for our numerical calculation (the precise value of $\kappa_{w}^{-1}$ is unimportant for the physics in oil discussed below). Inside the dielectric particle, the Poisson equation in the absence of external charges reads $\nabla^{2} \phi=0$. On the particle surface, we have the boundary condition $\mathbf{n} \cdot\left[\left.\epsilon_{c} \nabla \phi\right|_{\text {in }}-\left.\epsilon_{o} \nabla \phi\right|_{\text {out }}\right] /$ $\epsilon_{o}=4 \pi \lambda_{B}^{o} \sigma(\mathbf{r})$, with $\mathbf{n}$ an outward pointing normal vector and where $\sigma(\mathbf{r})$ follows from $\delta \Omega / \delta \sigma(\mathbf{r})=0$ [46,47], resulting for $\mathbf{r} \in \Gamma$ in the Langmuir adsorption isotherm [48], $\quad \sigma(\mathbf{r})= \pm \sigma_{m}\left(1+K_{ \pm} \exp \left\{ \pm\left[\phi(\mathbf{r})-\phi_{D}\right]\right\} / \rho_{s}^{o}\right)^{-1}$. Equation (2) is solved numerically for $\phi(\mathbf{r})$, from which $\rho_{ \pm}(\mathbf{r})$ and $\sigma(\mathbf{r})$ follow, and after insertion into Eq. (1) we find the particle-interface interaction Hamiltonian $H(d)$ := $\min _{\rho_{ \pm}, \sigma} \Omega\left[\rho_{ \pm}, \sigma ; d\right] . H(d)$ does not contain the particleinterface VDW repulsion, which can be added separately [45]. 
An example of the resulting potential distribution around a positively charged colloidal sphere can be found in Figs. 1(b) and 1(c), which reveals how $\phi(\mathbf{r})$ approaches its asymptotic value $\phi_{D}$ at $z \rightarrow \infty$ for various axial distances $r$ from the particle, revealing a strong coupling between the spherical and planar geometry of the particle and the interface, respectively. Since $f_{+}<f_{-}$(and hence $\phi_{D}>0$ ), the oil side is positively charged and the water side is negatively charged. This strong coupling is further illustrated in Fig. 2, where the (scaled) ion density is plotted for distances $d=10 a, 5 a$, and $1.5 a$, in (a)-(c) for a positively charged particle and in (d)-(f) for a negatively charged particle. Upon approaching the interface, the particle double layer deforms as ions are stripped by the water phase, since they dissolve better in water. As a consequence, the initially planar double layer at the water side strongly deforms as well. This double layer destruction was investigated earlier for a dense laterally averaged monolayer [12], but here we laterally resolve the spatial structure of the double layers for the first time, even with charge regulation taken into account.

Interestingly, the surface charge distributions $\sigma(\vartheta)$ of negative and positive charge-regulating particles, shown in the insets in Fig. 2, are not related by $\sigma(\vartheta) \leftrightarrow-\sigma(\vartheta)$ because the antisymmetry is broken by $f_{+} \neq f_{-}$. Here, $\vartheta$ is the polar angle defined in Fig. 1(a). For $d \gg \kappa_{o}^{-1}$, the particle double layer has spherical symmetry, and $\sigma(\vartheta)$ is constant. Close to the interface, however, we unravel an interplay between mass-action and image-charge effects, which for $f_{-}>f_{+}$enhance each other for positive particles and counteract each other for negative particles. The enhanced cation (reduced anion) concentration close to the interface enhances (reduces) $|\sigma(\vartheta)|$ at the south pole $\vartheta=\pi$ for positive (negative) particles by mass action. The image-charge effect, in contrast, is independent of the charge sign and allows charge-regulating particles to lower their electrostatic energy by increasing $|Z|$ when the particle approaches a medium with a higher dielectric constant. Indeed, close to the interface the dielectric effect dominates, and for negative particles the south pole is actually higher charged $(\vartheta=\pi)$ than the north pole $(\vartheta=0)$ - see the inset in Fig. 2(f) - in contrast to the case farther from the interface [Figs. 2(d) and 2(e)]. The interplay of mass action and dielectric effects is also seen in the total particle charge $Z=\int_{\Gamma} d^{2} \mathbf{r} \sigma(\mathbf{r})$, which depends on $d$ as shown in the insets in Fig. 3 for low and high $|Z|$ in (a) and (b), respectively. This is of interest not only for $\left(f_{+}, f_{-}\right)=(6,10)$, but also for $f_{+}=f_{-}=0$ (blue curves), to isolate the image-charge effects by switching off ion partitioning. Mass action thus increases (decreases) $|Z(d)|$ when $Z>0(Z<0)$, while dielectric effects always increase $|Z(d)|$. The image-charge effect is weak at low $|Z|$ but strong enough to drive negatively charged particles even more negative close to the interface. Combined with ion partitioning, this yields a minimum in $|Z(d)|$, if $Z$ is sufficiently negative [Fig. 3(b)].

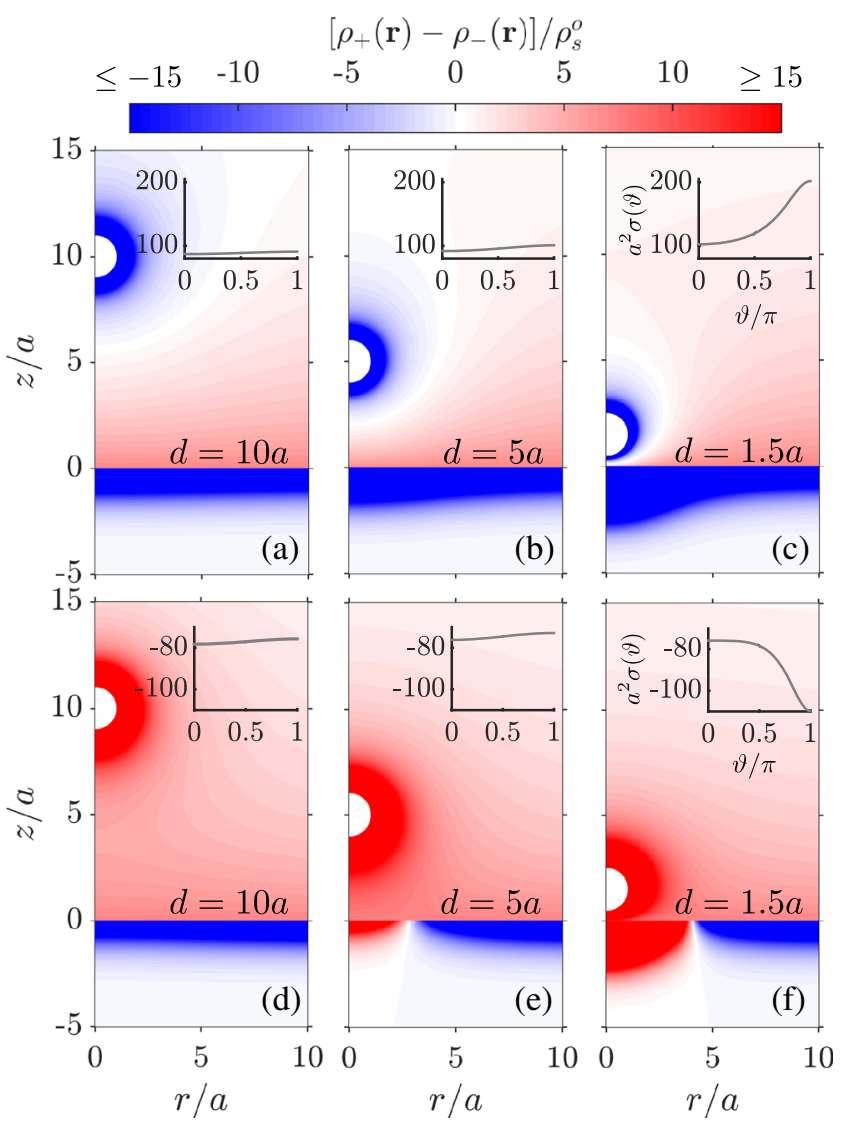

FIG. 2. Double layer destruction for a charged particle with radius $a=1 \mu \mathrm{m}$, with self-energies $\left(f_{+}, f_{-}\right)=(6,10)$, oil screening length $\kappa_{o}^{-1}=10 a$, equilibrium constant $K_{ \pm} a^{3}=1$ $\left(p K_{ \pm}=8.8\right)$, and bulk salt concentrations $\rho_{s}^{0}=0.093 \mathrm{nM}$ (oil) and $\rho_{s}^{w}=0.28 \mu \mathrm{M}$ (water). In (a)-(c) the particle is positively charged $(Z>0)$, and in (d)-(f) negatively charged $(Z<0)$. For clarity, we introduced a cutoff for the scaled net ion density $\left[\rho_{+}(\mathbf{r})-\rho_{-}(\mathbf{r})\right] / \rho_{s}^{o}$. The insets show the particle charge distribution $a^{2} \sigma(\vartheta)$.

In Fig. 3, we plot the particle-interface interaction potential $\Phi(d)=H(d)-H(\infty)$, which is repulsive for $Z<0$ (red curve) and attractive for $Z>0$ (green curve) provided that $f_{+}<f_{-}$. The blue curves, for $f_{+}=f_{-}=0$, show a much smaller $|\Phi(d)|$; i.e., the particle-interface interaction is dominated by ionic rather than image-chargelike effects. In Supplemental Material [45], we added the repulsive VDW potential to $\Phi(d)$ to show that the particles can indeed be trapped a few nanometers from the interface. Our calculations also reveal that it is possible to impede adsorption in the case of an attractive VDW interaction, since for $\phi_{D}>0$ and $Z<0$ (or as we shall see later also for $\phi_{D}<0$ and $Z>0$ ) there is a wetting-preventing energy barrier whose location and height can be tuned by the magnitude of $|Z|$ and/or $\phi_{D}$.

To further analyze the particle-interface interactions, we consider $\tilde{\Phi}(d)=Z(\infty) \phi_{0}(d)+\Phi_{\mathrm{di}}(d)$, with $\phi_{0}(z)$ the (analytically available) Gouy-Chapman-like electrostatic potential without any particle present [24] as plotted in 


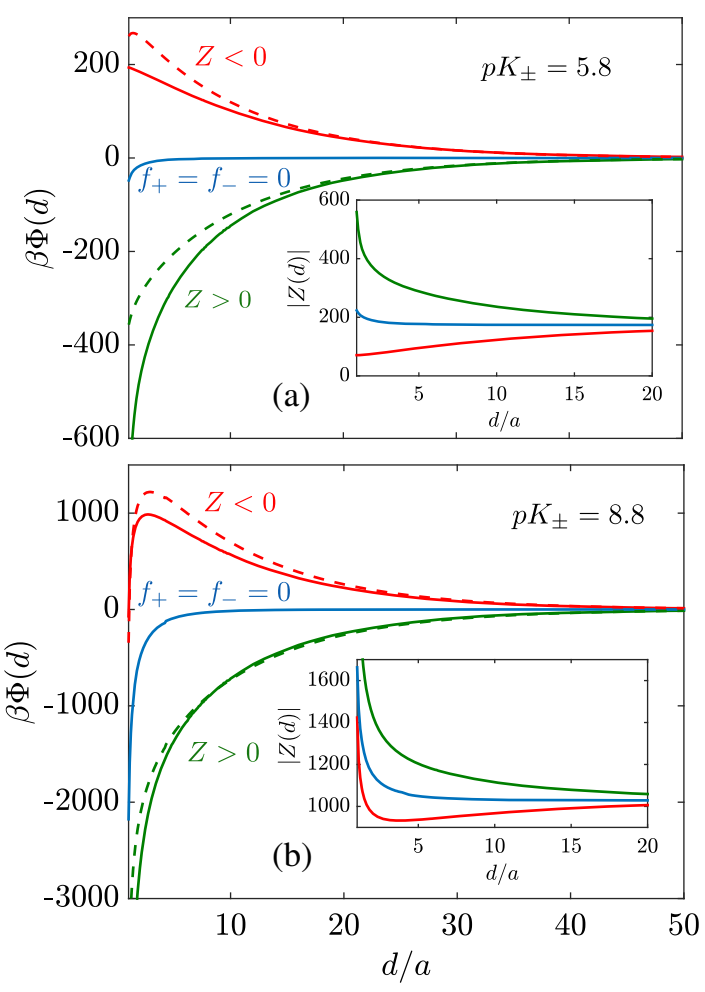

FIG. 3. Particle-interface potential $\Phi(d)$ for oil screening length $\kappa_{o}^{-1}=10 a$. The full curves are the results for (a) $p K_{ \pm}=5.8$ and (b) $p K_{ \pm}=8.8$. The red (green) curves are for a negative (positive) particle with self-energies $\left(f_{+}, f_{-}\right)=(6,10)$, and the blue curves are for $f_{+}=f_{-}=0$ and are independent of the sign of $Z$. The insets show the absolute charge $|Z(d)|$. The green $(Z>0)$ and red $(Z<0)$ dashed curves show the approximation $\tilde{\Phi}(d)$.

Fig. 1(b) [black solid line; note that $\phi_{0}(z \rightarrow \infty)=\phi_{D}$ ], and $\Phi_{\mathrm{di}}(d)=\left.\Phi(d)\right|_{f_{+}=f_{-}=0}$, the purely dielectric numerically obtained interaction potential. In this empirical approximation for $\Phi(d), \phi_{0}(d)$ acts as an external (electric) potential for a particle with charge $Z(\infty)$. The dashed green and red curves in Fig. 3 show that $\tilde{\Phi}(d)$ is a good approximation for $\Phi(d)$. Hence, $\kappa_{o}^{-1}$ is the relevant length scale for the particle-interface interaction, confirming that $\Phi(d)$ is insensitive to the precise value of $\kappa_{w}^{-1}$. It turns out that $\tilde{\Phi}$ approximates $\Phi$ for constant-charge particles perfectly if $|Z|$ is low [49].

To estimate whether $Z(\infty) \phi_{0}(d)$ or $\Phi_{\mathrm{di}}(d)$ is dominant, we equate both terms in absolute value at contact, $\left|Z(\infty) \phi_{0}(a)\right|=\Phi_{\mathrm{di}}(a)$, and approximate $\Phi_{\mathrm{di}}(a) \approx \Phi_{\mathrm{im}}(a)$ with $\epsilon_{w} \gg \epsilon_{o}$. The latter approximation gives an upper bound for the image-charge attractions, since salt will enlarge screening if no ion partitioning is included. We find that ion partitioning dominates image charges when $|Z(\infty)| \lambda_{B}^{o} / 4 a$ is small compared to $\left|\phi_{D}\right|$, as is the case in Fig. 3(a). In Fig. 3(b), we have $|Z(\infty)| \lambda_{B}^{o} / 4 a \sim\left|\phi_{D}\right|$, such that dielectric effects and ion partitioning are both important, although the dielectric effects are significant only for $d<\kappa_{o}^{-1}$. For $|Z(\infty)| \lambda_{B}^{o} / 4 a \gg\left|\phi_{D}\right|$, dielectric effects would dominate; however, it is hard to enter this regime experimentally, as it would require either finetuning the self-energies $f_{\alpha}$ to obtain extremely small Donnan potentials or particle charges exceeding $\mathcal{O}\left(10^{4}\right)$ in oil. Hence, the tunable Donnan potential is important in most experimental setups and can be measured [50]. Moreover, we note that changing the sign of $Z(\infty)$ is equivalent to interchanging $f_{+} \leftrightarrow f_{-}$, changing only the sign of $\phi_{D}$, showing that the nature of the particle-interface interactions can also be tuned by changing the type of salt. Finally, when multiple ions are included, $\phi_{D}$ will depend not only on the self-energies, but also on the bulk ion concentrations [24], extending even further the tunability options.

This Letter thus shows the importance of a low, but nonzero salt concentration in oil for the particle-interface interaction of an oil-dispersed particle. We showed that tuning the sign of the particle charge or Donnan potential can change attractive particle-interface interactions into repulsions. This sheds light on the very recent experimental studies by Elbers et al. [13], who showed not only that adding a salt with $f_{+}<f_{-}$to the oil changes $Z>0$ to $Z<0$, but also that particles are repelled from the interface to the bulk. Our calculations support this observation, since attractions become repulsions (Fig. 3) upon changing the sign of the particle charge, if a suitable charge regulation mechanism is provided. For example, a binary adsorption model in a three-ion system can be used, where global charge neutrality ensures that the cation and anion concentrations can be varied independently. Indeed, in the experiment of Ref. [13], there are three ions, namely, $\mathrm{H}^{+}$, $\mathrm{Br}^{-}$, and the organic tetrabutylammonium cation, and it is hypothesized that only $\mathrm{H}^{+}$and $\mathrm{Br}^{-}$can adsorb on the particle. Moreover, the range of the repulsion upon the inversion of the particle charge is of the order $\kappa_{o}^{-1}$, much larger than the range of the VDW repulsion that was proposed earlier as the responsible mechanism $[13,16]$. Our findings also have repercussions for a VDW attraction, since the Donnan potential impedes adsorption of oildispersed particles by a salt-tunable energy barrier for $Z(\infty) \phi_{D}<0$. Our work is relevant for designing tunable and reversible Pickering emulsions, which can be applied in drug delivery and food processing [51,52], but also for novel experiments where an ion flux can induce repulsive or attractive surface-specific interactions depending on the surface chemistry of the suspended particles [53].

We acknowledge fruitful discussions with N. Elbers, J. van der Hoeven, and A. van Blaaderen and financial support of a Netherlands Organisation for Scientific Research (NWO) VICI grant funded by the Dutch Ministry of Education, Culture and Science (OCW) and from the European Union's Horizon program under the Marie Skłodowska-Curie Grant Agreement No. 656327. 
This work is part of the D-ITP consortium, a program of the Netherlands Organisation for Scientific Research (NWO) funded by the Dutch Ministry of Education, Culture and Science $(\mathrm{OCW})$.

*j.c.everts@uu.nl

[1] P. Pieranski, Phys. Rev. Lett. 45, 569 (1980).

[2] S. U. Pickering, J. Chem. Soc., Trans. 91, 2001 (1907).

[3] M. E. Leunissen, A. van Blaaderen, A. D. Hollingsworth, M. T. Sullivan, and P. M. Chaikin, Proc. Natl. Acad. Sci. U.S.A. 104, 2585 (2007).

[4] M. Luo, G. K. Olivier, and J. Frechette, Soft Matter 8, 11923 (2012).

[5] F. Reincke, W. K. Kegel, H. Zhang, M. Nolte, D. Wang, D. Vanmaekelbergh, and H. Möhwald, Phys. Chem. Chem. Phys. 8, 3828 (2006).

[6] V. Poulichet and V. Garbin, Proc. Natl. Acad. Sci. U.S.A. 112, 5932 (2015).

[7] S. Crossley, J. Faria, M. Shen, and D. E. Resasco, Science 327, 68 (2010).

[8] B. O. Carter, W. Wang, D. J. Adams, and A. I. Cooper, Langmuir 26, 3186 (2010).

[9] B. O. Carter, D. J. Adams, and A. I. Cooper, Green Chem. 12, 783 (2010).

[10] M. E. Leunissen, J. Zwanikken, R. van Roij, P. M. Chaikin, and A. van Blaaderen, Phys. Chem. Chem. Phys. 9, 6405 (2007).

[11] C. P. Kelleher, A. Wang, G. I. Guerrero-García, A. D. Hollingsworth, R. E. Guerra, B. J. Krishnatreya, D. G. Grier, V. N. Manoharan, and P. M. Chaikin, Phys. Rev. E 92, 062306 (2015).

[12] J. Zwanikken and R. van Roij, Phys. Rev. Lett. 99, 178301 (2007).

[13] N. Elbers, J. van der Hoeven, M. de Winter, C. Schneijdenberg, M. van der Linden, L. Filion, and A. van Blaaderen, Soft Matter (to be published).

[14] See Supplemental Material at http://link.aps.org/ supplemental/10.1103/PhysRevLett.117.098002 for a few illustrative microscope pictures of the nontouching behavior of PMMA particles near a CHB-water interface taken from Ref. [13] (Fig. S1).

[15] See Supplemental Material at http://link.aps.org/ supplemental/10.1103/PhysRevLett.117.098002 for a few illustrative microscope pictures of the salt-induced dislodgment of PMMA particles near a CHB-water interface taken from Ref. [13] (Fig. S2).

[16] M. Oettel, Phys. Rev. E 76, 041403 (2007).

[17] J. D. Jackson, in Classical Electrodynamics, third ed. (Wiley, New York, 1998).

[18] H. Wang, V. Singh, and S. H. Behrens, J. Phys. Chem. Lett. 3, 2986 (2012).

[19] V. A. Parsegian, Van der Waals Forces: A Handbook for Biologists, Chemists, Engineers, and Physicists (Cambridge University Press, Cambridge, England, 2005).

[20] Y. Levin, J. Chem. Phys. 113, 9722 (2000).

[21] M. Bier, J. Zwanikken, and R. van Roij, Phys. Rev. Lett. 101, 046104 (2008).

[22] A. Onuki, J. Chem. Phys. 128, 224704 (2008).
[23] J. Zwanikken, J. de Graaf, M. Bier, and R. van Roij, J. Phys. Condens. Matter 20, 494238 (2008).

[24] M. J. E. Westbroek, N. Boon, and R. van Roij, Phys. Chem. Chem. Phys. 17, 25100 (2015).

[25] K. D. Danov, P. A. Kralchevsky, K. P. Ananthapadmanabhan, and A. Lips, Langmuir 22, 106 (2006).

[26] D. Frydel, S. Dietrich, and M. Oettel, Phys. Rev. Lett. 99, 118302 (2007).

[27] C. L. Wirth, E. M. Furst, and J. Vermant, Langmuir 30, 2670 (2014).

[28] R. R. Netz, Phys. Rev. E 60, 3174 (1999).

[29] A. Würger, Eur. Phys. J. E 19, 5 (2006).

[30] I. D. Morrison, Colloids Surf. A 71, 1 (1993).

[31] C. P. Royall, M. E. Leunissen, A.-P. Hynninen, M. Dijkstra, and A. van Blaaderen, J. Chem. Phys. 124, 244706 (2006).

[32] T. Kanai, N. Boon, P. J. Lu, E. Sloutskin, A. B. Schofield, F. Smallenburg, R. van Roij, M. Dijkstra, and D. A. Weitz, Phys. Rev. E 91, 030301 (2015).

[33] G. N. Smith, J. E. Hallett, and J. Eastoe, Soft Matter 11, 8029 (2015).

[34] M. N. van der Linden, J. C. P. Stiefelhagen, G. HeesselsGürboğa, J. E. S. van der Hoeven, N. A. Elbers, M. Dijkstra, and A. van Blaaderen, Langmuir 31, 65 (2015).

[35] M. Bier, A. Gambassi, M. Oettel, and S. Dietrich, Europhys. Lett. 95, 60001 (2011).

[36] M. Bier, A. Gambassi, and S. Dietrich, J. Chem. Phys. 137, 034504 (2012)

[37] A. Onuki, Phys. Rev. E 73, 021506 (2006).

[38] R. Okamoto and A. Onuki, Phys. Rev. E 84, 051401 (2011).

[39] S. Samin and Y. Tsori, Europhys. Lett. 95, 36002 (2011).

[40] A. P. dos Santos and Y. Levin, Langmuir 28, 1304 (2012).

[41] S. Samin and Y. Tsori, J. Chem. Phys. 139, 244905 (2013).

[42] S. Samin, M. Hod, E. Melamed, M. Gottlieb, and Y. Tsori, Phys. Rev. Applied 2, 024008 (2014).

[43] We neglect any possible interfacial deformations [44]. In the experiments of Ref. [13], no significant interfacial deformation was observed in a two-dimensional setup.

[44] B. J. Park, M. Lee, B. Lee, and E. M. Furst, Soft Matter 11, 8701 (2015).

[45] See Supplemental Material at http://link.aps.org/ supplemental/10.1103/PhysRevLett.117.098002 for the effect of a repulsive van der Waals interaction on $\Phi(d)$ (Fig. S3).

[46] B. Zoetekouw, Ph. D. thesis, Utrecht University, 2006.

[47] N. Boon and R. van Roij, J. Chem. Phys. 134, 054706 (2011).

[48] B. W. Ninham and V. Parsegian, J. Theor. Biol. 31, 405 (1971).

[49] See Supplemental Material at http://link.aps.org/ supplemental/10.1103/PhysRevLett.117.098002 for a comparison of a constant-charge boundary condition with the approximation $\tilde{\Phi}(d)$ (Fig. S4).

[50] M. Vis, V.F. D. Peters, R. H. Tromp, and B. H. Erne, Langmuir 30, 5755 (2014).

[51] Y. Chevalier and M.-A. Bolzinger, Colloids Surf. A 439, 23 (2013).

[52] J. Tang, P. J. Quinlan, and K. C. Tam, Soft Matter 11, 3512 (2015).

[53] A. Banerjee, I. Williams, R. N. Azevedo, M. E. Helgeson, and T. M. Squires, Proc. Natl. Acad. Sci. U.S.A. 113, 8612 (2016). 